\title{
The Role of the Vagal Response in Outcomes after Ablation for Atrial Fibrillation
}

\author{
Zeng $Y^{*}$, Meng $\mathbf{X}$, Li Y, Xu C and Han J
}

Atrial Fibrillation Center, Anzhen Hospital, Beijing, China

\begin{abstract}
Objective: The aim of this study was to assess the incremental benefit of denervation of vagal responses for the prevention of recurrence of atrial fibrillation (AF) in patients undergoing a mini-maze procedure.

Patients and methods: Between September 2007 and May 2009, 209 patients (aged $53 \pm 10$ years, 63 male) undergoing cardiac surgery underwent concomitant radiofrequency ablation for persistent AF. Intraoperative highfrequency stimulation showed a positive vagal response in 103 patients and a negative vagal response in 106 patients. The average history of AF was $36 \pm 43$ months. Preoperatively, 144 patients (68.9\%) were in New York Heart Association functional class III or IV.

Results: There were no in-hospital deaths, and no significant differences in mortality or complications between the two groups during the postoperative or follow-up periods. Immediately after surgery, 132 patients (63.2\%) were in sinus rhythm, with a similar proportion of sinus rhythm in the groups with positive and negative vagal responses (56.3\% vs. $62.3 \%, p=0.38$ ). At the latest follow-up (mean $18.9 \pm 9$ months), $60.8 \%$ of patients had freedom from $A F$, with a higher proportion of freedom from AF in patients with positive vagal responses than those with negative vagal responses $(68.2 \%$ vs. $53.3 \%, p=0.0004)$. Cox univariate and multivariate analyses identified positive vagal response as a predictor of late recurrence of $\mathrm{AF}$ (Wald=9.71;95\% Cl, $0.081-0.563 ; p=0.002)$. Logistic regression analysis showed that positive vagal response was inversely related to left atrial dimension (Wald $=4.45 ; 95 \% \mathrm{Cl}, 0.965-0.999$; $p=0.035)$. The proportion of patients with left atrial dimension $>70 \mathrm{~mm}$ was larger in the group with negative vagal responses than the group with positive vagal responses $(48.1 \%$ vs. $30.1 \%, p=0.008)$. Active ganglionated plexi were found in $49.2 \%$ of patients (102/209) with a mean number of $3.6 \pm 2.2$ sites (range 1-11) per patient. The majority of active sites (78.6\%) were located at the pulmonary vein (PV) antrum rather than the PV ostia, including $81.9 \%$ of right-sided sites and $70.5 \%$ of left-sided sites.

Conclusion: The results of this study indicate that parasympathetic attenuation by PV denervation achieved an incremental benefit in patients undergoing $\mathrm{PV}$ isolation for AF. In patients with persistent AF, positive vagal response was related to small left atrial diameter, and predicted outcome after ablation.
\end{abstract}

Keyword: Atrial fibrillation; Parasympathetic; High-frequency stimulation; Ablation

\section{Introduction}

Atrial fibrillation (AF) affects approximately 2.3 million people in North America and 4.5 million people in Europe, and approximately 10 million people in China [1,2]. Since Cox first reported that AF could be treated by surgical intervention, various modifications of the complex and time-consuming Cox maze III procedure have been reported [35]. Treatment for AF is evolving as understanding of the underlying mechanisms increases. Recent studies reported that parasympathetic autonomic tone plays an important role in triggering and maintaining AF [6]. Several studies reported significantly better outcomes in patients with a positive vagal response during catheter ablation and denervation than in patients with a negative vagal response [7-9]. The reasons for this remain unclear. Complete vagal denervation may result in a significant reduction in recurrence of AF in patients with a positive vagal response. It is unclear why some patients have a positive vagal response to stimulation and others have a negative response. Enlargement of the left atrium has been shown to be a strong predictor of recurrence of AF. It is unclear whether there is a relationship between the left atrial dimension and the vagal response. The findings of this study may help to clarify some of these issues.

\section{Patients and Methods}

\section{Patients}

Between September 2007 and May 2008, 209 patients (82 males) undergoing cardiac surgery underwent concomitant radiofrequency ablation for persistent AF. All patients gave written informed consent for inclusion in this study. The study protocol was approved by the Ethics Committee of the hospital. The patients were divided into two groups according to the results of intraoperative mapping of active ganglionated plexi: those with a positive vagal response to stimulation of ganglionated plexi (GP+ group, $n=103)$ and those with a negative vagal response to stimulation of ganglionated plexi (GP- group, $n=106$ ). Patients with a previous history of cardiac surgery or with left atrial thrombus on preoperative ultrasonography were excluded. Detailed preoperative data of the patients are shown in (Table 1). The average age of the patients was $52 \pm 11$ years (range 11-77 years). The preoperative New York Heart Association (NYHA) functional class was III or IV in 144 patients (68.9\%). The mean left atrial diameter

*Corresponding author: Zeng $\mathrm{Y}$, Section of Pharmacology, Department of Cardiology, Atrial Fibrillation Center, Anzhen Hospital, Beijing 100029, China, Tel: +86 10 63926382; E-mail: zengyaping2@163.com

Received June 29, 2014; Accepted September 22, 2014; Published September 29, 2014

Citation: Zeng Y, Meng X, Li Y, Xu C, Han J (2014) The Role of the Vagal Response in Outcomes after Ablation for Atrial Fibrillation. J Cardiovasc Dis Diagn 2: 176 doi:10.4172/2329-9517.1000176

Copyright: (๑) 2014 Zeng Y, et al. This is an open-access article distributed under the terms of the Creative Commons Attribution License, which permits unrestricted use, distribution, and reproduction in any medium, provided the original author and source are credited. 


\begin{tabular}{|c|c|c|c|}
\hline & GP + (103 cases) & GP- (106 cases) & P valve \\
\hline Age (years) & $53.6 \pm 10.1$ & $51.8 \pm 11$ & 0.23 \\
\hline Gender (F/M, \%) & $74.2 \%$ & $75.7 \%$ & 0.43 \\
\hline NYHA III-IV ( $\mathrm{n}, \%)$ & $75(70.8 \%)$ & $69(67 \%)$ & 0.56 \\
\hline History of thromboembolism & $13(12.6 \%)$ & $10(9.4 \%)$ & 0.46 \\
\hline Duration of AF (months) & $53 \pm 62$ & $58 \pm 61$ & 0.75 \\
\hline LA diameter >70 mm (n, \%) & $31(30.1 \%)$ & $51(48.1 \%)$ & $0.008^{\star}$ \\
\hline LVEDD (mm) & $50.6 \pm 8.1$ & $51.8 \pm 10.0$ & 0.31 \\
\hline LVEF $(\%)$ & $58.5 \pm 8.7$ & $60.4 \pm 8.6$ & 0.112 \\
\hline
\end{tabular}

Data are presented as number (percentage) of patients or mean \pm standard deviation. NYHA: New York Heart Association functional class, AF: atrial fibrillation, LA: left atrial, LVEDD: left ventricular end-diastolic dimension, LVEF: left ventricular ejection fraction.

${ }^{*} p<0.05$.

Table 1: Clinical characteristics of the patients in the two groups

was $68 \pm 16 \mathrm{~mm}$ (range $29-76 \mathrm{~mm}$ ) and the mean left ventricular ejection fraction was $59 \pm 9 \%$ (range $27-78 \%$ ). There was no significant difference in concomitant cardiac conditions between the two groups (Table 2).

\section{Preoperative procedures}

Baseline 12-lead electrocardiography (ECG) and chest X-ray were performed in all patients. Transthoracic and transesophageal echocardiography were performed to exclude left atrial thrombus. Warfarin was discontinued 3 days before surgery and the prothrombin time-international normalized ratio was confirmed to be in the normal range on the day before surgery. Antiarrhythmic drugs were continued.

\section{Surgical procedures}

All operations were performed under general anesthesia and cardiopulmonary bypass, using a radiofrequency (RF) generator system (AtriCure, OH, USA). The fat pad at the interatrial groove was resected by electrocautery before making a left atrial incision. Pulmonary vein (PV) isolation was performed, including a cuff of left atrial tissue and avoiding the PV ostia. It was fired four times, with repositioning each time to include more atrial cuff and fat pad tissues. The ablation procedure included lines encircling the right and left PVs, a line between the right and left superior PVs in the roof of the left atrium, and a line from the left inferior PV to the posterior mitral valve. The left atrial appendage was also excluded by external ligation or internal stitching, and the ligament of Marshall was ligated. Epicardial temporary pacing wires were routinely placed at the end of operation.

\section{Mapping}

Entrance block was considered successful if a baseline positive sensing result (rapid and disorderly atrial potentials) in the PV antrum before PV isolation was followed by a negative sensing result (no atrial potentials) in the same area after ablation. Positive baseline pacing was defined as presence of atrial capture, and negative post-ablation pacing was defined as absence of capture in the same area after ablation. Transmural block of conduction of the PV antrum was defined as achievement of both entrance and exit block. The left-sided procedure included additional techniques, such as division of the ligament of Marshall and excision or stitching of the left atrial appendage.

Mapping of active ganglionated plexi was performed before cardiopulmonary bypass by epicardial stimulation using a temporary pacemaker (Oscor Pace 203H DDD External Dual-Chamber Pacemaker; provided by Medtronic Company). Stimulation was performed at a maximum output of $18 \mathrm{~mA}$ and maximum rate of 1000 beats/min, following the sequence shown in (Figure 1). A positive response was defined as sinus bradycardia ( $\leq 40$ beats $/ \mathrm{min}$ ), asystole, atrioventricular block, or hypotension occurring within a few seconds of stimulation, coinciding with a decrease in blood pressure. We did not stimulate the next site until the blood pressure and heart rate had returned to normal. The interval between stimulations was at least $2 \mathrm{~min}$, to avoid depletion of neurotransmitters. Complete vagal denervation was defined as the abolition of all vagal responses. High-frequency burst stimulation was repeated in areas where active ganglionated plexi were initially identified. If any vagal responses remained, these areas were further locally ablated until all responses to high-frequency stimulation were considered negative as defined by no significant increase in the R-R interval during stimulation.

\section{Postoperative management and follow-up}

Intravenous amiodarone $(1 \mathrm{mg} / \mathrm{min})$ was administered to all patients for $6 \mathrm{~h}$, followed by continuous intravenous infusion $(0.5 \mathrm{mg} /$ $\mathrm{min}$ ) until oral intake was tolerated. Oral amiodarone (200-600 mg per day depending on heart rate) was administered for 6 months. Directcurrent cardioversion was performed in patients with recurrence of $\mathrm{AF}$ lasting $>12 \mathrm{~h}$ during the perioperative period or follow-up period. Daily 12-lead ECGs were performed during the hospital stay. All patients were followed up in the outpatient clinic at 1,3 , and 6 months after surgery. Twelve-lead ECG and transthoracic echocardiography was performed at each follow-up visit. Patients with symptoms suggesting arrhythmia were evaluated by ECG or 24 -h Holter monitor. The ECGs were mailed or faxed to a physician who was blinded to the procedure, for interpretation.

\section{Statistical analysis}

Continuous data are expressed as the mean \pm standard deviation, and proportions are expressed as percentages. Comparisons between the two groups were made using the Student's unpaired $t$ test for

\begin{tabular}{|c|c|c|c|}
\hline & $\begin{array}{l}\mathrm{GP}+(103 \\
\text { cases })\end{array}$ & GP- (106 cases) & $P$ value \\
\hline \multicolumn{4}{|l|}{ Procedure } \\
\hline MVR & $90(87.4 \%)$ & $89(84 \%)$ & 0.48 \\
\hline MVP & $7(6.8 \%)$ & $12(11.3 \%)$ & 0.255 \\
\hline AVR & $26(24.5 \%)$ & $29(28.2 \%)$ & 0.552 \\
\hline MVR+AVR & $2(1 \%)$ & $0(0)$ & 0.9 \\
\hline CABG & $14(6.3 \%)$ & $9(4.3 \%)$ & 0.67 \\
\hline ASD & $6(2.7 \%)$ & $2(0.9 \%)$ & 0.42 \\
\hline \multicolumn{4}{|l|}{ Concomitant procedure } \\
\hline TVP & $61(59.2 \%)$ & $58(54.7 \%)$ & 0.38 \\
\hline TVR & $9(8.7 \%)$ & $7(6.6 \%)$ & 0.32 \\
\hline Left atrial thrombectomy & $18(17.5 \%)$ & $14(13.2 \%)$ & 0.39 \\
\hline Left atrial folding & $5(2.4 \%)$ & $1(1.6 \%)$ & 0.46 \\
\hline \multicolumn{4}{|l|}{ Valve } \\
\hline Mechanical valve & $93(90.3 \%)$ & $92(86.6 \%)$ & 0.43 \\
\hline Bioprosthetic valve & $2(1.9 \%)$ & $1(0.9 \%)$ & 0.54 \\
\hline Ablation time (seconds) & $472.5 \pm 283.5$ & $520.8 \pm 284.3$ & 0.56 \\
\hline $\begin{array}{l}\text { Cardiopulmonary bypass } \\
\text { time (minutes) }\end{array}$ & $110.4 \pm 37.1$ & $114.8 \pm 43.2$ & 0.96 \\
\hline $\begin{array}{l}\text { Aortic cross-clamp time } \\
\text { (minutes) }\end{array}$ & $72.5 \pm 28.5$ & $72.3 \pm 23.8$ & 0.09 \\
\hline
\end{tabular}

Data are presented as number (percentage) of patients or mean \pm standard deviation. MVR: mitral valve replacement, MVP: mitral valve repair, AVR: aortic valve replacement, CABG: coronary artery bypass grafting, TVP: tricuspid valve repair, TVR: tricuspid valve replacement, CPB: cardiopulmonary bypass, ACC: aortic cross-clamping.

${ }^{*} p<0.05$ 

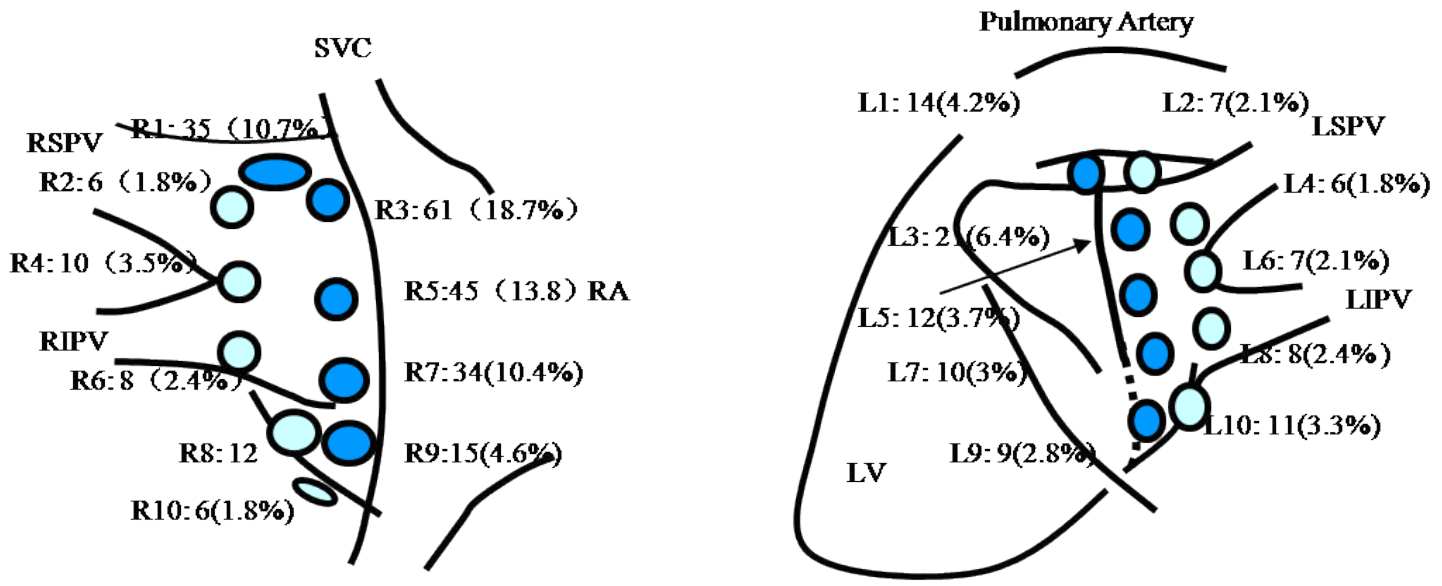

Figure 1: Diagram of epicardial mapping locations, and distributions of active ganglionated plexi before ablation. Ten right atrial sites (R1-R10) and 10 left atrial sites (L1-L10) were mapped. IVC: inferior vena cava, L: left, LA: left atrium, LIPV: left inferior pulmonary vein, LSPV: left superior pulmonary vein, LV: left ventricle, R: right, RA: right atrium, RIPV: right inferior pulmonary vein, RSPV: right superior pulmonary vein, SVC: superior vena cava.

\begin{tabular}{|c|c|c|}
\hline & GP+ (103 cases) & GP- (106 cases) \\
\hline Incision infection & $12(5.4 \%)$ & $8(7.5 \%)$ \\
\hline Renal failure & $2(1.9 \%)$ & $4(3.8 \%)$ \\
\hline Respiratory failure & $0(0)$ & $1(0.4 \%)$ \\
\hline Tracheotomy & $3(2.9 \%)$ & $3(2.8 \%)$ \\
\hline IABP & $3(2.9 \%)$ & $3(2.8 \%)$ \\
\hline ECMO & $3(2.9 \%)$ & 0.21 \\
\hline Reexploration for bleeding & $7(6.8 \%)$ & 0.97 \\
\hline pacemaker & 0 & $5(4.9 \%)$ \\
\hline
\end{tabular}

IABP: intra-aortic balloon pump, ECMO: extracorporeal membrane oxygenation.

Table 3: Complications in the groups with active ganglionated plexi (GP+) and without active ganglionated plexi (GP-).

continuous variables and the $\chi^{2}$ test for categorical variables. Freedom from recurrence of AF was estimated by the Kaplan-Meier method, and differences between groups were analyzed using the log-rank test. Univariate and multivariate Cox regression analyses were used to analyze the risk of late recurrence of $\mathrm{AF}$, and logistic regression analysis was used to analyze the relationship between active ganglionated plexi and left atrial dimension. Calculation of $95 \%$ confidence intervals was performed. The results were considered significant at $p<0.05$. All data were analyzed using SPSS 13.0 (SPSS Inc., Chicago, IL, USA).

\section{Results}

\section{Mortality and complications}

There were no hospital deaths in either group. During the followup period, one patient in the GP+ group died from a brain hemorrhage at 12 months after surgery, and one patient in the GP- group died from malfunction of a mechanical mitral valve. There were no significant differences in the rates of mortality and complications between the two groups during the postoperative and follow-up periods (Table 3 ).

\section{Stroke rate}

There were no cases of stroke during the perioperative period. During the follow-up period, one patient in the GP+ group who was taking warfarin because he had a mechanical mitral valve died from died from a brain hemorrhage at 12 months after surgery. There was no significant difference in the stroke rate between the two groups $(p=0.31)$.

\section{Mapping of ganglionated plexi}

Nearly half of the 209 patients $(103 / 209,49.2 \%)$ had at least one site where intraoperative high-frequency stimulation of ganglionated plexi resulted in a positive vagal response. A total of 327 active sites were identified, with a mean number of $3.6 \pm 2.2$ sites (range 1-11) per patient. These sites were all right-sided in $52 / 103$ patients $(50.5 \%)$, bilateral in $43 / 103$ patients $(41.7 \%$ ), and all left-sided in $8 / 103$ patients (7.8\%). The majority of active sites $(257 / 327,78.6 \%)$ were at the PV antrum rather than the PV ostia, including $81.9 \%$ (190/232) of rightsided sites and $70.5 \%$ (67/95) of left-sided sites. The details of the sites and numbers are shown in (Figure 1).

\section{Cardiac rhythm}

Immediately after surgery, sinus rhythm was observed in 132 patients $(63.2 \%)$, including $58(56.3 \%)$ in the GP+ group and 66 $(62.3 \%)$ in the GP- group ( $p=0.38)$; junctional rhythm in 41 patients (19.6\%), including $27(26.2 \%)$ in the GP+ group and $22(20.8 \%)$ in the GP- group ( $p=0.35)$; AF in 20 patients $(9.6 \%)$, including $11(10.7 \%)$ in the GP+ group and $9(8.5 \%)$ in the GP-group $(p=0.59)$; and paced rhythm in 16 patients $(7.7 \%)$, including $7(6.8 \%)$ in the GP+ group and $9(8.5 \%)$ in the GP- group $(p=0.65)$. At discharge, sinus rhythm was observed in 159 patients (76.1\%), including $79(76.7 \%)$ in the GP+ group and $80(75.5 \%)$ in the GP- group ( $p=0.85)$; junctional rhythm in 8 patients $(3.8 \%)$, including $4(3.9 \%)$ in the GP+ group and $4(3.8 \%)$ in the GP- group ( $p=0.97)$; $\mathrm{AF}$ in 29 patients $(13.9 \%)$, including 14 $(13.6 \%)$ in the GP+ group and $15(14.2 \%)$ in the GP- group $(p=0.91)$; and paced rhythm in 2 patients (1.0\%), including $1(1.0 \%)$ in the GP+ 
group and $1(1.0 \%)$ in the GP- group $(\mathrm{p}=0.05)$. The rhythms at the time of aortic declamping and at discharge in the two groups are shown in (Figure 2). Thirty-three patients (80\%) with a junctional rhythm at the time of aortic declamping had converted to sinus rhythm at discharge. Postoperative transient atrial flutter occurred during hospitalization in seven patients, and was not significantly different between the two groups.

The mean follow-up period was $18.9 \pm 9$ months (range 6-34 months) after surgery. Five patients in the GP+ group and 10 in the GP- group were lost to follow-up. Freedom from AF was observed in $67 / 98$ patients $(68.4 \%)$ in the GP+ group and $51 / 96$ patients $(53.3 \%)$ in the GP-group ( $p=0.037)$. In the GP+ group, ECG at the latest followup showed sinus rhythm in 62 patients $(63.3 \%)$, paced rhythm in 1 patient (1\%), AF in 31 patients (31.6\%), paroxysmal supraventricular tachycardia in 1 patient (1\%), atrial tachycardia in 1 patient (1\%), and atrial flutter in 2 patients (2\%). In the GP- group, ECG at the latest follow-up showed sinus rhythm in 49 patients (51\%), AF in 45 patients (47\%), paced rhythm in 2 patients (2\%), and atrial flutter in 4 patients (4.2\%). Freedom from recurrence of $\mathrm{AF}$ was estimated using the Kaplan-Meier method (Figure 3).

\section{Predictors of late recurrence of AF}

We analyzed the relationships between preoperative clinical characteristics and freedom from recurrence of AF. Multivariate analysis showed that left atrial dimension and active ganglionated plexi were predictors of recurrence of AF during the follow-up rhythm at aortic declamping

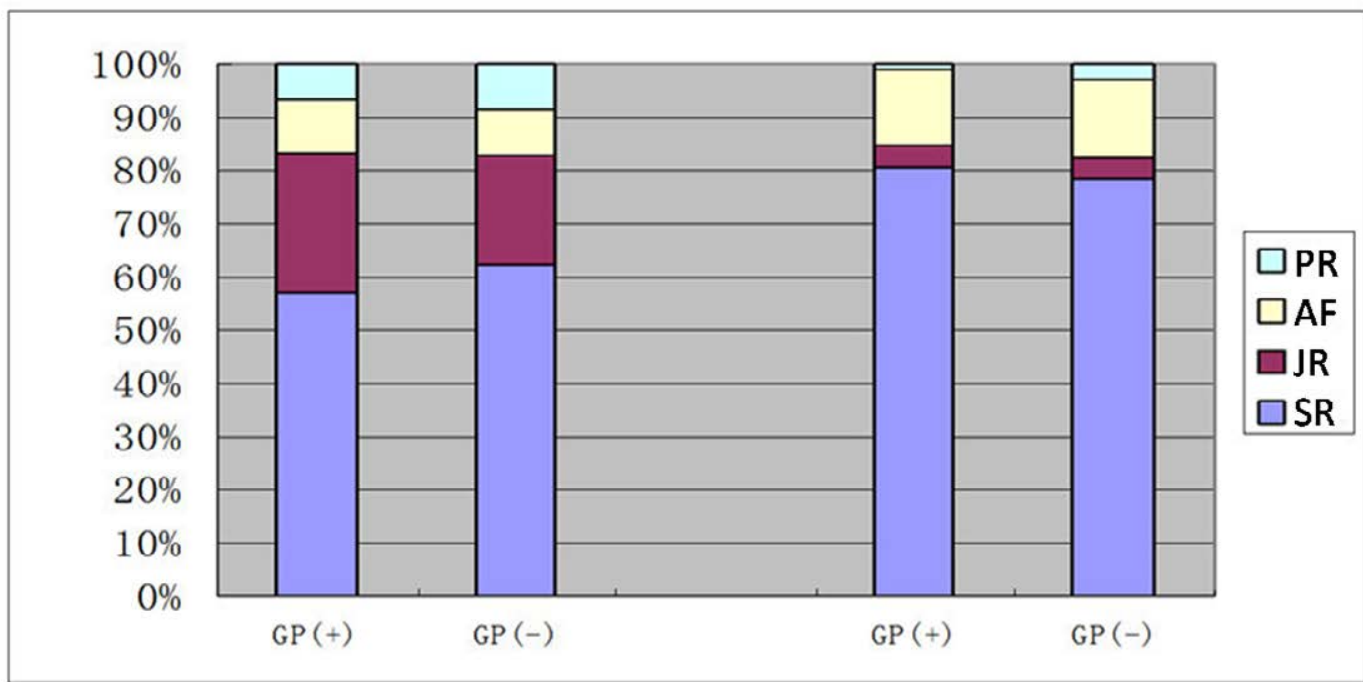

Figure 2: Rhythm at the time of aortic declamping and at discharge in patients with and without active ganglionated plexi. SR: sinus rhythm, NR: nodal rhythm, AF: atrial fibrillation, PR: paced rhythm.

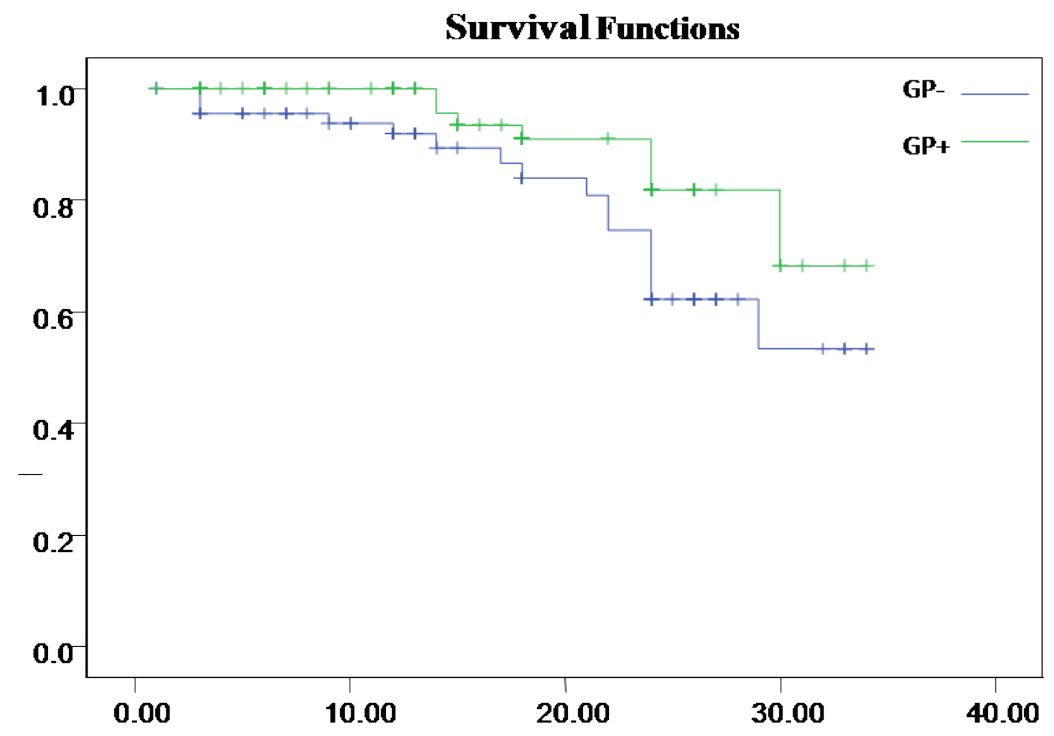

Figure 3: Freedom from recurrent atrial fibrillation was estimated in patients with and without active ganglionated plexi group using the Kaplan-Meier method. 
period. Left atrial dimension was identified as a significant predictor of outcome (Wald=13.746; 95\% CI, 1.016-1.053; $p<0.0001$ ). The left atrial dimension was larger in patients with recurrence of AF than in those without recurrence of AF. Active ganglionated plexi was also a significant predictor of recurrence of AF (Wald $=4.454$; 95\% CI, 0.965 $0.999 ; p=0.037$ ). Univariate and multivariate logistic regression analysis showed that the presence of active ganglionated plexi was inversely related to small left atrial diameter ( $95 \%$ CI, $0.965-0.999 ; p=0.035)$. The proportion of patients with large left atrial diameter was higher in the GP- group than in the GP+ group ( $48.1 \%$ vs. $30.1 \%, p=0.008)$.

\section{Postoperative clinical and echocardiographic findings}

Echocardiography was performed before and after surgery in all patients. Before surgery, 144 patients $(69 \%)$ were in NYHA functional class III or IV. At the latest follow-up, 155 patients (80\%) were in functional class I and 32 patients (17\%) were in functional class II. The NYHA functional class improved after surgery in all patients $(1.3 \pm$ 0.4 preoperatively vs. $2.8 \pm 0.5$ postoperatively, $p=0.006$ ). There was no significant difference in postoperative left atrial dimension between the $\mathrm{GP}+$ group and the GP- group $(54.3 \pm 15.3 \mathrm{~mm}$ vs. $51.7 \pm 16.9 \mathrm{~mm}$, $p=0.33$ ).

\section{Discussion}

$\mathrm{AF}$ is frequently associated with underlying heart disease. Surgical methods of treating AF have recently been developed. The goals of treatment are to (1) restore sinus rhythm and relieve AF-related symptoms, (2) reduce the occurrence of embolism due to AF, (3) reduce the impact of $\mathrm{AF}$ on cardiac function, and (4) restore atrial transport function. Based on these goals, the Cox maze III procedure and a number of modified mini-maze procedures have been developed for the surgical treatment of AF [10-13]. The outcomes are encouraging.

In this study, nodal rhythms were common after aortic declamping, occurring in $16 \%$ of patients, and $80 \%$ of these reverted to sinus rhythm by the time of discharge. The possible reasons for this are as follows. (1) Long-term rapid AF suppresses sinus node function, which takes time to recover $[14,15]$. (2) Denervation-based treatment affects sinus node function, which is affected by cardiac autonomic innervation. (3) Myocardial edema secondary to cardiopulmonary bypass and surgery affects sinus node function. Most nodal rhythms can convert to sinus rhythm and are considered benign.

Rhythm is affected by the PV neural anatomy. Wolf ${ }^{15}$ reported that $50 \%$ of patients with AF have active ganglionated plexi along the interatrial groove and the ligament of Marshall. Onorati et al. [16] reported that 16 of 75 patients had active ganglionated plexi bilaterally, 8 had only right-sided active ganglionated plexi, and 7 had only left-sided active ganglionated plexi, demonstrated by both electrophysiological and histological findings. Another study reported that the PV antrum within $5 \mathrm{~mm}$ of the PV ostia was more densely innervated than the proximal atria or the more distal parts of the PVs. Active ganglionated plexi are more dense near the superior right PV than the inferior right PV, and near the right PVs than the left PVs. Our findings are consistent with those reports, showing unilateral right-sided active ganglionated plexi in the majority of patients (54\%), especially at positions R1, R3, and R5 near the right superior PV, and bilateral active ganglionated plexi in $37.9 \%$ of patients. The majority of active ganglionated plexi were in the PV antrum (78.6\%). Some or all of the following factors may explain why ablation of the PV antrum is superior to PV isolation alone. (1) Isolation of the PV ostia can eliminate dormant ectopic foci in the PVs. (2) Re-entry may occur at the junction between the left atrium and the PVs, resulting in maintenance of AF [17]. This is consistent with intraoperative mapping findings that the quick disordered potentials at the PVs disappear after ablation of the PV antrum but not after PV isolation alone. (3) Ablation of the PV antrum can exclude $25-30 \%$ of the posterior left atrial wall, which plays a dormant role in maintaining AF [18]. (4) The risk of PV stenosis is significantly lower after ablation of the PV antrum than after isolation of the PV ostia. (5) Ablation of the PV antrum is the optimal procedure for modifying autonomic innervation, as shown in the present series.

Over the past several decades, treatment for AF has evolved as our understanding of the underlying mechanisms has increased. The processes of focal activation and conduction of fibrillation have been demonstrated [19]. Recent studies reported that parasympathetic autonomic tone plays an important role in triggering and maintaining AF. A synergistic relationship between active ganglionated plexi and ectopic PV foci may lower the threshold for induction of AF and increase the vulnerability of the atria to fibrillation $[18,20]$. Platt et al. [21] first tried to evaluate ablation of active ganglionated plexi as a stand-alone strategy for the treatment of AF, without changing the properties of the PVs. In patients with preoperative paroxysmal and chronic AF, sinus rhythm was maintained in $84 \%$ after 6 months of postoperative follow-up. It has also been reported that denervation alone guided by elicited vagal responses was a feasible method of treating AF [22]. In patients with positive vagal responses, those who underwent complete denervation according to high-frequency stimulation results experienced a significantly lower rate of recurrence of AF than those who did not undergo complete denervation. It remains unclear why only some patients have positive vagal responses, and why those with positive vagal responses have better outcomes than those with negative vagal responses. It is unknown whether there are mechanical reasons for these different outcomes. There is currently no strong evidence that left atrial enlargement is a predictor of recurrence of AF, and it is unclear whether there is a relationship between left atrial dimension and vagal responses. The aim of this study was to analyze the differences between patients in the GP+ and GP- groups. Our findings provided some information that may be useful for increasing our understanding of the role of the autonomic nervous system in AF, including: (1) Patients with positive vagal responses had better outcomes after ablation than those with negative vagal responses, which is consistent with the findings of previous studies $[23,24]$. (2) Left atrial dimension and active ganglionated plexi are predictors of recurrence of AF on univariate analysis and Cox multivariate regression analysis. Positive vagal responses are associated with smaller left atrial dimension. Active ganglionated plexi are an index of left atrial remodeling in patients with AF. Patients with active ganglionated plexi may have some unknown mechanisms that make it easy to control AF by ablating the relevant foci. (3) The following theories of cardiac autonomic nerve remodeling may explain the underlying molecular mechanism. (i) Ganglionated plexi may play a role in patients with both positive and negative vagal responses, but may be more active in those with positive vagal responses. (ii) High-frequency stimulation is a limited method of identifying active ganglionated plexi, and may miss active ganglionated plexi in some areas [25]. (iii) Stimulation of the autonomic reflex only occurs when the vagal nerve neurotransmitter acetylcholine binds to its receptor. (iv) AF results in atrial electrophysiological remodeling, anatomical remodeling, and cardiac autonomic nervous system remodeling. Mapping of active ganglionated plexi may provide a new index of left atrial remodeling. (v) Cardiac autonomic nervous system remodeling manifests as down-regulation of expression of acetylcholine receptors during AF [26]. (vi) The density of acetylcholine receptors decreases 
as the left atrium enlarges. In the present study of patients with AF, those with negative vagal responses had larger left atrial dimensions and more complex substrates than those with positive vagal responses. In patients with active ganglionated plexi, isolation of the PV antrum and denervation can easily remove the main foci triggering fibrillation and can therefore achieve better outcomes. Isolation of the PV antrum in patients without active ganglionated plexi is less likely to eliminate all the foci and therefore has poorer outcomes.

\section{Limitations}

This is a retrospective study, and a large-scale prospective study is still required. Changes in autonomic nervous system activity are reflected by the heart rate. We did not assess autonomic nervous system activity, such as low-frequency and high-frequency R-R variability and the low-to-high frequency ratio. The theory of down-regulation of acetylcholine receptors still requires large-scale histological evidence. The different mechanisms underlying $\mathrm{AF}$ in patients with positive and negative vagal responses also need further investigation.

\section{Conclusions}

The results of mapping of active ganglionated plexi can be used as an index of left atrial remodeling in patients with AF, and can be used to predict outcomes after ablation for AF. Active ganglionated plexi are more common at the PV antrum than the PV ostia, suggesting that ablation of the PV antrum is reasonable and can achieve complete denervation. In patients with positive vagal responses, denervation can achieve an incremental benefit when undergoing PV ablation for persistent AF.

\section{References}

1. Fuster V, Rydén LE, Cannom DS, Crijns HJ, Curtis AB, et al. (2006) American Heart Association, European Society of Cardiology. ACC/AHA/ESC 2006 guidelines for the management of patients with atrial fibrillation-executive summary. J Am Coll Cardiol 48: 854-906.

2. Zhou ZQ, Hu DY, Chen J, Zhang RH, Li KB, et al. (2004) An epidemiological survey of atrial fibrillation in China. Chin J Intern Med 43: 491-494.

3. Schuessler RB, D'Agostino HJ, Stone CM, Chang BC, Cain ME, et al. (1991) The surgical treatment of atrial fibrillation. Development of a definitive surgical procedure. J Thorac Cardiovasc Surg 101: 569-583.

4. Kainuma S, Yokota T, Toda K, Taniguchi K (2009) Modified maze procedure combined with mitral valve surgery via septal-superior approach. Eur J Cardiothorac Surg 36: 198-199.

5. 5. Nitta T, Lee R, Watanabe H, Harris KM, Erikson JM, et al. (1999) Radia approach: a new concept in surgical treatment for atrial fibrillation. II. Electrophysiologic effects and atrial contribution to ventricular filling. Ann Thorac Surg 67: 36-50.

6. Coumel P (1996) Role of the autonomic nervous system in paroxysmal atria fibrillation. In: Touboul PC, Waldo AL (Eds) Atrial Flutter. Armonk, Futura Publishing Co, USA.

7. Schauerte P, Scherlag BJ, Pitha J, Scherlag MA, Reynolds D, et al. (2000) Catheter ablation of cardiac autonomic nerves for prevention of vagal atrial fibrillation. Circulation 102: 2774-2780.

8. Scherlag BJ, Nakagawa H, Jackman WM, Yamanashi WS, Patterson E, et al. (2005) Electrical stimulation to identify neural elements on the heart: their role in atrial fibrillation. J Intervent Card Electrophysiol 13: 1-6.
9. Pappone C, Santinelli V, Manguso F, Vicedomini G, Gugliotta F, et al. (2004) Pulmonary vein denervation enhances long-term benefit after circumferential ablation for paroxysmal atrial fibrillation. Circulation 109: 327-334.

10. Stulak JM, Sundt TM 3rd, Dearani JA, Daly RC, Orsulak TA, et al. (2007) Tenyear experience with the Cox-maze procedure for atrial fibrillation: how do we define success? Ann Thorac Surg 83: 1319-1325.

11. Fasol R, Meinhart J, Binder T (2005) A modified and simplified radiofrequency ablation in patients with mitral valve disease. J Thorac Cardiovasc Surg 129: 215-217.

12. Cui YQ, Sun LB, Li Y, Xu CL, Han J, et al. (2008) Intraoperative modified Cox mini-maze procedure for long-standing persistent atrial fibrillation. Ann Thorac Surg 85: 1283-1289.

13. Gaynor SL, Diodato MD, Prasad SM, Ishii Y, Schuessler RB, et al. (2004) A prospective, single-center clinical trial of a modified Cox maze procedure with bipolar radiofrequency ablation. J Thorac Cardiovasc Surg 128: 535-542.

14. Elvan A, Pride HP, Eble JN, Zipes DP (1995) Radiofrequency catheter ablation of the atria reduces inducibility and duration of atrial fibrillation in dogs. Circulation 91: 2235-2244.

15. Mehall JR, Kohut RM, Schneeberger W, Taketani T, Merrill WH, et al. (2007) Intraoperative epicardial electrophysiologic mapping and isolation of autonomic ganglionic plexi. Ann Thorac Surg 83: 538-541.

16. Onorati F, Curcio A, Santarpino G, Torella D, Mastroroberto P, et al. (2008) Routine ganglionic plexi ablation during Maze procedure improves hospital and early follow-up results of mitral surgery. J Thorac Cardiovasc Surg 136: 408418.

17. Dong JZ, Liu XP (2006) Maintenance mechanism of Atrial Fibrillation-“a pulmonary vein-left atrium reentry" hypothesis. Adv Cardiovasc Dis 27: 9-12.

18. Pappone C, Santinelli V (2005) Atrial fibrillation ablation: state of the art. Am J Cardiol 96: 59-64.

19. Nitta T, Ishii Y, Miyagi Y, Ohmori H, Sakamoto S, et al. (2004) Concurrent multiple left atrial focal activations with fibrillatory conduction and right atrial focal or reentrant activation as the mechanism in atrial fibrillation. $\mathrm{J}$ Thorac Cardiovasc Surg 127: 770-778.

20. Lemola K, Chartier D, Yeh YH, Dubuc M, Cartier R, et al. (2008) Pulmonary vein region ablation in experimental vagal atrial fibrillation: role of pulmonary veins versus autonomic ganglia. Circulation 117: 470-477.

21. Platt M, Mandapati R, Scherlag BJ (2004) Limiting the number and extent of radiofrequency app lications to terminate atrial fibrillation and subsequently prevent its inducibility. Heart Rhythm 1: 11.

22. Pokushalov E, Turov A, Shugayev P, Artyomenko S, Romanov A, et al. (2008) Catheter ablation of left atrial ganglionated plexi for atrial fibrillation. Asian Cardiovasc Thorac Ann 16: 194-201.

23. Pappone C, Santinelli V, Manguso F, Vicedomini G, Gugliotta F, et al. (2004) Pulmonary vein denervation enhances long-term benefit after circumferential ablation for paroxysmal atrial fibrillation. Circulation 109: 327-34.

24. Scanavacca M, Pisani CF, Hachul D, Lara S, Hardy C, et al. (2006) Selective atrial vagal denervation guided by evoked vagal reflex to treat patients with paroxysmal atrial fibrillation. Circulation 114: 876-885.

25. Onorati F, Curcio A, Santarpino G, Torella D, Mastroroberto P, et al. (2008) Routine ganglionic plexi ablation during Maze procedure improves hospital and early follow-up results of mitral surgery. J Thorac Cardiovasc Surg 136: 408418

26. Sarmast F, Kolli A, Zaitsev A, Parisian K, Dhamoon AS, et al. (2003) Cholinergic atrial fibrillation: I(K,Ach) gradients determine unequal left/right atrial frequencies and rotor dynamics. Cardiovasc Res. 59: 863-873. 